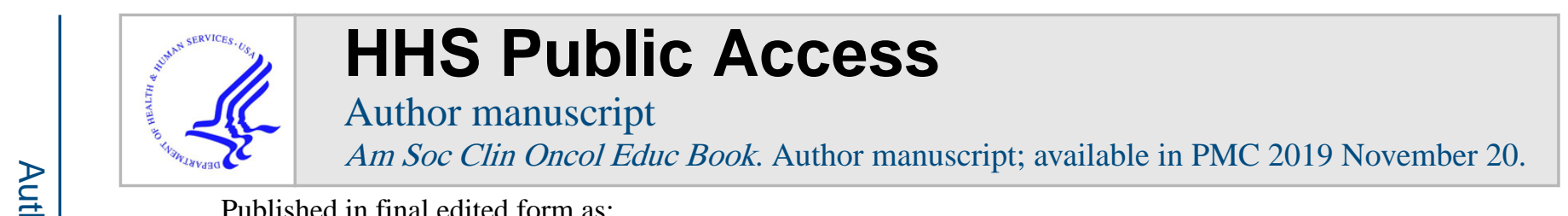

Published in final edited form as:

Am Soc Clin Oncol Educ Book. 2018 May 23; 38: 330-341. doi:10.1200/EDBK_201215.

\title{
Personalized Management of Advanced Kidney Cancer
}

\author{
Jeffrey Graham, MD, Daniel Y. C. Heng, MD, James Brugarolas, MD, PhD, Ulka \\ Vaishampayan, MD \\ Tom Baker Cancer Centre, University of Calgary, Calgary, Alberta, Canada; Kidney Cancer \\ Program, Simmons Comprehensive Cancer Center, University of Texas Southwestern Medical \\ Center, Dallas, TX; Karmanos Cancer Institute, Wayne State University, Detroit, MI.
}

\section{OVERVIEW}

The treatment of renal cell carcinoma represents one of the great success stories in translational cancer research, with the development of novel therapies targeting key oncogenic pathways. These include drugs that target the VEGF and mTOR pathways, as well as novel immuno-oncology agents. Despite the therapeutic advancements, there is a paucity of well-validated prognostic and predictive biomarkers in advanced kidney cancer. With a number of highly effective therapies available across multiple lines, it will become increasingly important to develop a more tailored approach to treatment selection. Prognostic clinical models, such the International Metastatic Renal Cell Carcinoma Database Consortium (IMDC) model, are routinely used for prognostication in clinical practice. The IMDC model has demonstrated a predictive capability in the context of these treatments including immune checkpoint inhibition. A number of promising molecular markers and gene expression signatures are being explored as prognostic and predictive biomarkers, but none are ready to be widely used for treatment selection. In this review, we will explore the current landscape of personalized care in metastatic renal cell carcinoma. This will include a focus on both prognostic and predictive factors as well as clinical applications of biology in kidney cancer.

The treatment of renal cell carcinoma (RCC) has undergone a dramatic evolution over the last decade. The improvements in treatment are secondary to a better understanding of the biologic factors driving cancer growth. The elucidation of the importance of the VEGF and mTOR pathways led to the introduction of several novel agents in the treatment of metastatic renal cell carcinoma (mRCC). More recently, several new immuno-oncology agents have shown impressive activity in advanced kidney cancer and are currently being explored in combination with targeted therapy. ${ }^{1}$ The evolution of targeted therapy as the mainstay of management in RCC has been a dominant part of the advances.

Despite these impressive successes in exploiting molecular targets, there has been a paucity of biomarkers in RCC that can predict response or clinical outcomes with the novel agents. As the number of therapeutic options increases, it is critical to develop a personalized strategy to treatment, taking into consideration both tumor and patient characteristics to

Corresponding author: Ulka Vaishampayan, MD, Karmanos Cancer Center, Wayne State University, 4100 John R St., Detroit, MI 48201; vaishamu@karmanos.org.

Disclosures of potential conflicts of interest provided by the authors are available with the online article at asco.org/edbook. 
develop a tailored treatment plan. In a disease such as RCC where the spectrum of overall survival (OS) ranges from a few months to many years even without administration of any systemic therapy, the risk prognostication of the patients is of paramount importance in therapeutic decision making. Individualized care using predictive biomarkers is central to the treatment of other advanced malignancies. This includes the anti-HER2 antibody in HER2amplified breast cancer, anti-EGFR therapies in $K R A S$ wild-type colorectal cancer, and BRAF inhibitors in BRAF mutant melanomas. ${ }^{2}$ Thus, the elucidation of predictive factors is an unmet need in mRCC and an area of active research. ${ }^{3}$

In this review, we will explore the current landscape of personalized care in mRCC. This will include a focus on both prognostic and predictive factors as well as clinical applications of biology in kidney cancer. We will provide examples of a personalized approach to systemic therapy and explore future directions in the individualized treatment of advanced kidney cancer.

\section{OVERVIEW OF PROGNOSTIC CLINICAL FACTORS}

A personalized approach to the treatment of cancer necessitates an understanding of the variables influencing prognosis. Prior to the advent of targeted agents, a commonly used prognostic risk index was the Memorial Sloan Kettering Cancer Center model. This model was developed and validated in the era of interferon therapy, and it incorporated a number of clinical and biochemical variables. The Memorial Sloan Kettering Cancer Center model integrated five adverse factors: Karnofsky performance status of less than $80 \%$, elevated lactate dehydrogenase, high corrected serum calcium, low hemoglobin, and interval from diagnosis to treatment of less than 1 year. Based on the number of pretreatment factors, three prognostic groups were identified: favorable (zero risk factors), intermediate (one to two risk factors), and poor risk (more than two risk factors). ${ }^{4}$

The IMDC prognostic model was developed in the more modern era of VEGF-targeted therapy. In total, six variables were identified as having prognostic significance in this population. ${ }^{5}$ These included two clinical factors (a Karnofsky performance status of less than $80 \%$ and time from diagnosis to initiation of therapy of less than 1 year) as well as four laboratory factors (hemoglobin below the lower limit of normal and elevated corrected calcium, neutrophil count, and platelet counts greater than the upper limit of normal). The final IMDC model was able to successfully stratify real-world patients into three distinct prognostic groups: favorable (zero risk factors), intermediate (one to two risk factors), and poor risk (more than two risk factors). Table 1 summarizes the Memorial Sloan Kettering Cancer Center and IMDC prognostic models.

The IMDC model was externally validated using a cohort of 1,028 real-world patients from 13 international cancer centers. In this analysis, the median OS associated with each prognostic group was 43 months, 23 months, and 8 months in the favorable, intermediate, and poor risk groups, respectively. ${ }^{6}$ The IMDC model continues to be widely used to stratify patients in contemporary clinical trials and to provide personalized, risk-directed treatment selection in everyday clinical practice. The recent trials of nivolumab, cabozantinib, and 
ipilimumab and nivolumab as well as lenvatinib and everolimus have used the IMDC criteria, as they were specifically applicable to anti-VEGF therapy with sunitinib.

Since the initial validation of the IMDC model, it has been studied in a number of other populations of patients with RCC. Similar to the first-line setting, the IMDC model has been demonstrated to provide prognostic stratification in both the second and third-line settings.

7,8 Because the original IMDC model included predominately clear cell renal cell carcinoma (ccRCC), Kroeger et $\mathrm{al}^{9}$ examined the applicability of the IMDC prognostic model in advanced non-clear cell renal cell carcinoma (nccRCC). In this population, patients with nccRCC had inferior OS (12.8 vs. 22.3 months) compared with patients with ccRCC. Similar to the clear cell population, the IMDC model was able to reliably stratify the nccRCC cohort into three distinct prognostic groups. ${ }^{9}$ More recently, the IMDC was shown to provide prognostic stratification among patients receiving second-line immunotherapy agents, including the immune checkpoint inhibitor nivolumab. ${ }^{10}$

Given that the IMDC prognostic model did not include patients receiving pazopanib, PerezValderrama et a ${ }^{11}$ conducted a retrospective observational study to validate the model in this population. The study included 278 patients treated with first-line pazopanib for $\mathrm{mRCC}$ in 34 centers in Europe. Within this cohort, 19.4\% had favorable risk, $57.2 \%$ had intermediate risk, and $23.4 \%$ had poor risk. As with first-line sunitinib, the IMDC model was able to estimate the prognosis of patients treated with first-line pazopanib. The median OS was not reached in the favorable risk group and was 21.6 months and 7.1 months in the intermediate and poor risk groups, respectively.

Beyond the aforementioned variables included in the IMDC model, there have been a number of other clinical factors demonstrated to have prognostic significance in advanced kidney cancer. These include the baseline neutrophil-to-lymphocyte ratio (NLR) and the presence of bone and liver metastases. McKay et al ${ }^{12}$ examined the prognostic impact of bone and liver metastases in a retrospective analysis of 2,027 patients with $\mathrm{mRCC}$ treated with first-line targeted therapy. Both of these factors were associated with inferior outcomes, with hazard ratios (HR) of 1.38 and $1.37(\mathrm{p}<.0001)$ for the presence of bone and liver metastases, respectively. Elevated markers of systemic host inflammation, such as NLR, have been shown to be associated with a poor prognosis in several solid tumors. Templeton et $\mathrm{al}^{13}$ explored the impact of baseline NLR on survival in advanced RCC. In this analysis, higher NLR at baseline was associated with shorter OS (adjusted HR per 1-unit increase in $\operatorname{lnNLR}, 1.69 ; 95 \% \mathrm{CI}, 1.46-1.95 ; \mathrm{p}<.001) .{ }^{13}$

Although uncovering variables associated with poor prognosis is important in developing personalized treatment strategies, identifying factors that do not influence clinical outcomes is also helpful. As the overall life expectancy of our population increases, understanding the impact of age on cancer outcomes will become increasingly important. Khambati et al ${ }^{14}$ explored the use of first-line targeted therapy in elderly patients (older than age 75) with mRCC. In this analysis, outcomes were found to be similar between the older and younger subgroups, even when adjusted for known poor prognostic factors. These findings suggest that age alone should not be used as an absolute contraindication to targeted therapy. 


\section{OBSERVATION/SURVEILLANCE OF THE PATIENT WITH METASTATIC RENAL CELL CARCINOMA}

$\mathrm{mRCC}$ is a heterogeneous disease and one that is characterized by a variable natural history. There appears to be a certain subset of patients with $\mathrm{mRCC}$ who may display a less aggressive and more indolent pattern of progression. Given these observations, there has been ongoing interest in the idea of deferred systemic therapy with active surveillance, in contrast to the more standard approach of starting therapy immediately at the onset of metastatic disease. This deferred approach has been examined in a number of retrospective analyses as well as prospectively in a large observational registry, the Metastatic Renal Cell Cancer Registry. ${ }^{15,16}$ Park et al ${ }^{17}$ performed a retrospective analysis of 58 patients undergoing active surveillance for mRCC. In this series, the median time to disease progression was 12.4 months. Multivariable analysis revealed that Karnofsky performance status of less than $100 \%$, liver metastases, and time from diagnosis to the start of surveillance of less than 1 year were associated with a shorter time to progression. Importantly, the response rate and OS for the subsequent systemic treatment after surveillance were comparable with those of previous reports.

Rini et al ${ }^{18}$ conducted a prospective phase II trial designed to examine the feasibility and safety of an initial active surveillance approach in the era of modern targeted therapy. They included patients with asymptomatic mRCC, measurable disease, and no prior systemic therapy. In total, 52 patients were enrolled into the study. Patients were radiographically assessed at baseline and then every 3 months for year 1, every 4 months for year 2 , then every 6 months thereafter. The decision to initiate systemic therapy was at the discretion of the treating physician and patient. The median surveillance time until initiation of systemic therapy was 14.9 months. Multivariate analysis showed that a higher number of IMDC risk factors $(\mathrm{p}=.0403)$ and a greater number of metastatic sites $(\mathrm{p}=.0414)$ were associated with a shorter surveillance period. Based on this, the authors identified a favorable subgroup, defined as patients with zero to one IMDC risk factor and fewer than two organs with metastatic disease, who had an estimated median surveillance time of 22.2 months. These findings suggest that deferred initiation of systemic therapy using an active surveillance protocol may be appropriate for carefully selected patients with low-risk disease.

\section{MANAGEMENT OF OLIGOMETASTATIC RENAL CELL CARCINOMA}

Despite improvements in systemic therapy options, local therapy to sites of metastatic disease remains an important component in the personalized management of mRCC. These therapies can include surgical resection (metastasectomy), definitive radiotherapy, and other ablative procedures. The most common sites of metastatic disease in RCC are the lung $(45 \%)$, bone (30\%), lymph nodes (20\%), liver (20\%), adrenal gland (9\%), and brain (8\%). ${ }^{19}$ For each of these sites, there is evidence that local therapies may be effective, particularly in the setting of a limited number of metastases. In a series of 141 patients treated with metastasectomy in the pretargeted therapy era, curative intent resection was associated with a 44\% 5-year OS rate. ${ }^{20} \mathrm{~A}$ disease-free interval greater than 12 months from the time of 
nephrectomy, solitary site (vs. multiple sites) of metastasis, and age younger than 60 were associated with improved survival.

With regard to resection of pulmonary metastases, Pfannschmidt et $\mathrm{al}^{21}$ retrospectively analyzed 191 patients with pulmonary metastases from RCC who underwent surgical resection. The 5-year survival rate after complete metastasectomy was $41.5 \%$. Favorable prognostic factors included having fewer than seven metastatic lesions and a disease-free interval of greater than 23 months. Pancreatic metastases also tend to have a favorable outcome after resection. ${ }^{22}$ Solitary bone and soft tissue metastases should also be considered for local therapy. Embolization of metastases, especially bone metastasis, prior to resection is strongly advised to reduce the risk of hemorrhage and complications.

The role of local therapy in the management of RCC is rapidly increasing. Noninvasive techniques such as stereotactic radiation therapy or cryotherapy are increasingly being applied for oligometastatic disease and for consolidative control of residual masses after systemic therapy. ${ }^{23}$ Aoun et $\mathrm{al}^{24}$ reported on cryoablation of more than 2,000 tumor masses including metastatic sites and renal masses and established efficacy of the procedure. The successful experience with a series of patients specifically with advanced kidney cancer treated with cryotherapy has helped establish the safety, feasibility, and efficacy of this procedure. Wang et $\mathrm{al}^{23}$ evaluated stereotactic ablative radiation therapy for extracranial RCC metastases and reported results on 175 metastatic foci, where they observed 1-year local control rates in excess of $90 \%$. There are also emerging data on abscopal effects and of synergy between immunotherapy and ablative techniques as a result of the release of neoantigens. ${ }^{25}$ Currently, clinical trials evaluating the direct and abscopal clinical effects and immune changes with a combination of radiation or cryotherapy and immune checkpoint inhibition are in development.

The role of systemic therapy following complete resection of metastatic disease is unclear. The ECOG 2810 phase III trial is comparing adjuvant pazopanib with placebo after metastasectomy and may help clarify the use of targeted agents in resected mRCC. Other trials exploring immune checkpoint inhibitors also allow the inclusion of patients with completely resected metastatic disease, such as KEYNOTE-564, which compares pembrolizumab with placebo in the adjuvant setting, or IMmotion010, which evaluates atezolizumab.

\section{CLINICAL APPLICATION OF PROGNOSTIC AND PREDICTIVE FACTORS IN FRONTLINE THERAPY OF METASTATIC RENAL CELL CARCINOMA}

In the first-line setting, there are a number of established therapeutic options in mRCC. These include VEGF-targeted drugs (e.g., sunitinib, pazopanib, and cabozantinib), mTOR inhibitors, high-dose interleukin (IL)-2, and more recently, immuno-oncology agents. ${ }^{1}$ One of the cornerstones of personalized care in oncology is the discovery and validation of factors that can predict response to various therapeutic agents. These can include clinical or patient-specific factors as well as tumor-specific biomarkers. In this section, we will review clinical factors that may be used to help establish a more personalized approach to the treatment of advanced RCC. 
Prognostic clinical factors are important in guiding treatment decisions in mRCC. In 2007, Hudes et $\mathrm{a}^{26}$ conducted a phase III randomized trial exploring the role of the mTOR inhibitor temsirolimus in previously untreated, poor-risk, advanced RCC. In this trial, 626 patients were randomly assigned to temsirolimus, temsirolimus plus interferon-alfa, or interferon-alfa monotherapy. Inclusion criteria necessitated that patients have at least three of the following six predictors of poor prognosis: elevated lactate dehydrogenase, elevated serum calcium, low hemoglobin, time from diagnosis to randomization of less than 1 year, Karnofsky performance status of 60 or 70, and metastases in multiple organs. In this poorrisk population, temsirolimus significantly prolonged the median OS compared with interferon-alfa as a single agent (10.9 vs. 7.3 months; HR for mortality, 0.73; 95\% CI, 0.580.92). These results led the U.S. Food and Drug Administration to approve temsirolimus in the first-line setting for patients with a poor prognosis. Although this trial did not directly compare the use of an mTOR inhibitor among patients with good-intermediate versus poor risk, it did provide a risk-directed approach to treatment selection. It is important to note that the prognostic index used in this trial is different from the IMDC model. In real-world practice, the use of temsirolimus in this setting is limited as a result of intravenous administration.

Risk profiling has also been used in studies using VEGF-targeted agents. In the recently reported phase II CABOSUN trial, 157 patients with intermediate or poor risk mRCC based on IMDC criteria were randomly assigned to cabozantinib or sunitinib. ${ }^{27}$ Cabozantinib is a VEGF, MET, and AXL inhibitor. Patients were stratified by IMDC risk category (intermediate or poor) and presence of bone metastases. The response rate for cabozantinib was $46 \%$, with a significantly increased median progression-free survival (PFS; 8.2 vs. 5.6 months; HR 0.66; 95\% CI, 0.46-0.95; $\mathrm{p}=.012$ ) compared with sunitinib. OS was also increased with cabozantinib, (26.6 vs. 21.2 months), but the difference was not statistically significant (HR 0.79; 95\% CI, 0.53-1.20). A PFS benefit with cabozantinib was also observed in subgroup analyses, including among those with poor IMDC risk and bone metastases. The results of the independent review of response and PFS in the CABOSUN trial led the U.S. Food and Drug Administration to approve cabozantinib in the frontline setting of RCC. Table 2 summarizes the key results of the CABOSUN trial.

More recently, the predictive capability of the IMDC model was demonstrated in the CheckMate 214 clinical trial. ${ }^{28}$ This was a large phase III randomized controlled trial evaluating the combination of two immune checkpoint inhibitors, nivolumab and ipilimumab, compared with standard sunitinib alone in previously untreated advanced RCC or mRCC. This trial stratified patients by IMDC prognostic group and sample size was powered for overall analysis and for the intermediate and poor risk groups. In the IMDC intermediate and poor risk groups, combination immunotherapy was associated with superior OS compared with sunitinib (HR 0.63; $\mathrm{p}$.0001). In contrast, the IMDC favorable group appeared to have superior outcomes with sunitinib alone in terms of response rate and PFS. In the favorable risk group, the objective response rate (ORR) was $52 \%$ versus $29 \%$ (p $=.0002)$ and PFS was 25.2 versus 15.3 months (HR 2.18; $\mathrm{p}$ <.0001) in the sunitinib and immunotherapy arms, respectively. These data suggest that favorable risk tumors may have a distinct biology, which is characterized by greater dependence on VEGF signaling. 
The investigators also performed an exploratory analysis looking at PD-L1 expression. Patients with a tumor PD-L1 of $1 \%$ or greater demonstrated a higher response rate and improved PFS with nivolumab and ipilimumab compared with sunitinib, but those with less than $1 \%$ expression demonstrated a response rate of $37 \%$. This suggests that PD-L1 expression may increase the likelihood or response but cannot accurately predict which patients will not respond. With regard to toxicity, an increased incidence of high-grade treatment-related adverse events was observed with sunitinib. This may have been influenced by the early introduction of steroids, which were required for $60 \%$ of patients with combination immunotherapy. Health-related quality of life data were also collected, with patients reporting better symptom control with immunotherapy versus sunitinib. It is unclear why the intermediate and poor risk groups appeared to benefit more from immunotherapy. This may be attributable to higher levels of neoantigen exposure and immune stimulation or secondary to unidentified biomarkers common to this group. Tumors in the intermediate and poor risk groups may also be more inflamed, which may account for increased neutrophil or platelet counts as well as anemia and possibly a worse performance status. The results of this pivotal trial have the potential to transform the treatment landscape of mRCC, and they provide clinicians with a more personalized approach to upfront systemic therapy. Table 3 summarizes the key results of the CheckMate 214 trial.

Other regimens that are currently in ongoing phase III trials consist of a common theme of comparing combinations of anti-VEGF therapy and immunotherapy, using sunitinib as the control arm. One of the combinations, bevacizumab and atezolizumab, has shown favorable outcomes in the PD-L1-positive population in a randomized trial (IMmotion 151) compared with the control arm of sunitinib.

\section{Role of Cytoreductive Nephrectomy in Metastatic Renal Cell Carcinoma}

Selecting patients with advanced kidney cancer that may benefit from a cytoreductive nephrectomy $(\mathrm{CN})$ is another important decision in the personalized treatment of this population. In the pretargeted therapy era, a combined analysis of two prospective randomized clinical trials revealed that $\mathrm{CN}$ followed by interferon treatment was associated with a 5.8-month increase in OS versus interferon alone (13.6 vs. 7.8 months). ${ }^{29}$ Unfortunately, there has been a lack of randomized trials exploring the role of $\mathrm{CN}$ for patients with $\mathrm{mRCC}$ being treated with molecularly targeted therapy. To investigate this further, a retrospective analysis was performed to address the survival benefit of $\mathrm{CN}$ for patients with mRCC treated with targeted therapy. ${ }^{30}$ The median OS for patients with CN versus without $\mathrm{CN}$ was 20.6 versus 9.5 months ( $\mathrm{p}<.0001$ ). When adjusted for IMDC risk criteria to correct for imbalances, the HR of death was 0.60 (95\% CI, 0.52-0.69; p <.0001). Importantly, patients who possessed four or more of the IMDC prognostic factors did not appear to benefit from CN. Thus, not all patients should be offered this procedure and the decision should be individualized based on prognosis. In the real world, other considerations include bulk of tumor burden outside of the kidney, brain/liver metastases, symptoms from the primary tumor, and surgical feasibility.

In the recently reported SURTIME trial, investigators attempted to determine whether the sequence of $\mathrm{CN}$ among patients who receive sunitinib has an effect on patient outcomes. ${ }^{31}$ 
In this trial, patients with metastatic ccRCC were randomly assigned to immediate $\mathrm{CN}$ followed by sunitinib versus three cycles of sunitinib followed by $\mathrm{CN}$ plus sunitinib (deferred $\mathrm{CN}$ ). As a result of poor accrual, the investigators decided to report the progression-free rate at week 28 as the primary endpoint. No significant difference between the two groups was observed; the progression-free rate was $42.0 \%$ (95\% CI, 28.2-56.8) versus $42.9 \%$ (95\% CI, 28.8-57.8) in the immediate and deferred arms, respectively ( $\mathrm{p}>$. 99). Although the study was not adequately powered, an OS improvement was seen for deferred $\mathrm{CN}$. The authors concluded that the deferred approach initiates therapy quickly, does not lead to the inability to perform $\mathrm{CN}$, and $\mathrm{CN}$ after sunitinib appears to be safe.

\section{On-Treatment Predictors of Response}

Given the relative lack of well-validated predictive biomarkers, there has been interest in examining the use of on-treatment predictors of response. Most of these rely on mechanismbased adverse events that may act as a surrogate for clinical efficacy, focusing mainly on VEGF-targeted therapies. This class of drugs has specific toxicities, many of which have been analyzed as potential surrogate biomarkers. These include hypertension, hypothyroidism, neutropenia, thrombocytopenia, and hand-foot syndrome. ${ }^{32}$

The most well established of these adverse events is treatment-related hypertension. ${ }^{33}$ This side effect is common with sunitinib, occurring in approximately one-third of patients. ${ }^{34}$ Rini et a ${ }^{35}$ examined the association between sunitinib-induced hypertension and antitumor efficacy in a large retrospective, pooled analysis of four studies that included 544 patients with mRCC. In this study, hypertension was defined as a systolic blood pressure of at least $140 \mathrm{mmHg}$ or a diastolic blood pressure of at least $90 \mathrm{mmHg}$. Systolic hypertension was associated with an ORR of $54.8 \%$ compared with an ORR of $8.7 \%$ for patients without systolic hypertension ( $\mathrm{p}<.001)$. PFS (12.5 vs. 2.5 months; $\mathrm{p}<.001)$ and OS (30.9 vs. 7.2 months; $\mathrm{p}<.001)$ were also significantly longer for patients with systolic hypertension than for those without. Importantly, the incidence of hypertension-associated end organ dysfunction, such as cardiovascular events, was low. Although retrospective in nature, these findings suggest that treatment-induced hypertension may be a viable predictive biomarker of efficacy in mRCC. Other studies suggest that the phenomenon is generalizable to other anti-VEGF receptor therapies. Inasmuch as the hypertension is likely related to the magnitude of systemic VEGF receptor blockade, higher rates of hypertension may reflect higher effective drug levels.

\section{CLINICAL APPLICATION OF PROGNOSTIC AND PREDICTIVE FACTORS IN SECOND-LINE THERAPY AND BEYOND OF RENAL CELL CARCINOMA}

In the second-line setting, there are again a number of potential treatment options. These include targeted drugs such as axitinib, cabozantinib, lenvatinib plus everolimus, and the checkpoint inhibitor nivolumab. The optimal sequence of therapy is still an area of active research. Al-Marrawi et $\mathrm{al}^{36}$ used the IMDC data set to retrospectively investigate the association of clinical outcome between two lines of targeted therapy. In this study, 464 patients who received both a first- and second-line VEGF inhibitor were included. In this analysis, there was no correlation between first- and second-line responses, in terms of both 
ORR and PFS. For the entire group, the ORR for first-line therapy was $22 \%$ compared with $11 \%$ for the second-line therapy. These results suggest that in clinical practice, a patients response, or lack thereof, should not necessarily influence the choice of second-line targeted therapy.

The phase III METEOR trial helped establish cabozantinib as an effective second-line option in mRCC. ${ }^{37}$ In this trial, 658 previously treated patients were randomly assigned to receive cabozantinib (60 mg/day) or everolimus $(10 \mathrm{mg} /$ day $)$. All patients had progressed after receiving prior VEGF tyrosine kinase inhibitor therapy; $69 \%$ of patients had received only one prior course of systemic therapy, whereas $31 \%$ had been treated with two or more prior regimens. Patients progressing while treated with immune checkpoint inhibitors were also included. Randomization was stratified according to the number of previous VEGF tyrosine kinase inhibitors and prognostic risk category. Cabozantinib was associated with an improved PFS compared with everolimus (7.4 vs. 3.8 months), with a corresponding HR of 0.58 (95\% CI, 0.45-0.75; $\mathrm{p}<.001$ ). This PFS benefit was consistently observed in prespecified subgroups defined according to the number of prior VEGF tyrosine kinase inhibitors and prognostic risk category. A dedicated subset analysis of patients with bone metastases also revealed improved outcomes with cabozantinib. ${ }^{38}$ In this subgroup, the PFS was 7.4 months for cabozantinib versus 2.7 months for everolimus (HR 0.33; 95\% CI, 0.21 0.51). Median OS was also longer with cabozantinib (20.1 vs. 12.1 months; HR 0.54; $95 \%$ CI, 0.34-0.84). These results are consistent with those from the previously discussed CABOSUN trial and suggest that cabozantinib is an effective treatment option for this specific patient population.

Another recently approved second-line option in $\mathrm{mRCC}$ is the immune checkpoint inhibitor nivolumab. In the CheckMate 025 trial, 821 patients were randomly assigned to nivolumab ( $3 \mathrm{mg} / \mathrm{kg}$ every 2 weeks) or everolimus ( $10 \mathrm{mg} / \mathrm{day}$ ), with all patients having received one or two prior antiangiogenic therapies. ${ }^{39}$ Randomization was stratified by the prognostic risk group and the number of previous antiangiogenic therapy regimens. Nivolumab was associated with a significant improvement in OS (25.0 vs. 19.6 months; HR 0.73). The ORR was also greater with nivolumab compared with everolimus ( $25 \%$ vs. $5 \%$ ). Expression of PD-L1 on tumor cells was not associated with a survival benefit to nivolumab, because those with $1 \%$ or greater expression and those with less than $1 \%$ expression had a similar survival benefit compared with everolimus. Among patients with $1 \%$ or greater PD-L1 expression, the median OS was 21.8 months (95\% CI, 16.5-28.1) in the nivolumab group and 18.8 months (95\% CI, 11.9-19.9) in the everolimus group (HR 0.79; 95\% CI, 0.53-1.17). Higher levels of PD-L1 expression appeared to be associated with shorter OS irrespective of treatment arm. A substantial improvement in quality of life was also observed over the 2year study period during nivolumab treatment. A dedicated subgroup analysis of this trial has also been reported. This has confirmed a benefit to nivolumab across a number of key baseline factors, including risk groups, age, number and sites of metastases, and type and duration of prior therapy. ${ }^{40}$ Duration of response to prior anti-VEGF therapy and NLR (cutoff greater than or less than three) were noted to be independent predictors of benefit from nivolumab therapy in pretreated $\mathrm{RCC} .{ }^{41}$ In the context of immune therapy, no clear predictive or prognostic factors have emerged that have been correlated in large populations. 
Exploration of the IMDC database to evaluate prognostic criteria with immunotherapy treatment is ongoing.

\section{CLINICAL APPLICATIONS OF BIOLOGY IN RENAL CELL CARCINOMA}

The treatment of RCC represents one of the great success stories in translational cancer research, with the development of novel therapies targeting key oncogenic pathways. Despite these advancements, there is a relative lack of well-validated prognostic and predictive molecular biomarkers in advanced kidney cancer.

Broadly speaking, RCC represents a diverse collection of distinct histologic subtypes, with ccRCC comprising more than $75 \%$ of cases. ${ }^{42}$ Molecular heterogeneity within these subtypes is likely playing an important role in the diversity of responses and resistance to targeted therapies and has complicated biomarker discovery. ${ }^{2}$ The elucidation and subsequent clinical validation of these molecular markers will be critical in the development of a more personalized approach to treatment. In this section, we will highlight the contemporary clinical applications of biology in RCC. We will focus on potential molecular prognostic biomarkers as well as predictive factors related to VEGF-targeted therapy and immune checkpoint inhibitors.

\section{Prognostic Biomarkers}

Advancements in modern genomic techniques, including next-generation sequencing, have revealed the diverse spectrum of both genetic and epigenetic changes in kidney cancer. The most commonly mutated gene in ccRCC is von Hippel Lindau (VHL), which in inactivated in more than $50 \%$ of patients with ccRCC. ${ }^{43}$ This gene resides on chromosome 3 p25 and is essential to the regulation of hypoxia-inducible factor a and angiogenesis. Three other tumor suppressor genes (PBRM1, SETD2, and BAP1) are also located on chromosome 3p and together constitute the most frequently mutated genes after VHL. ${ }^{44}$ All three of these genes appear to be involved in regulating epigenetic processes such as chromatin and histone modification. ${ }^{2}$ Interestingly, whereas mutations in PBRM1 and $B A P 1$ tend to be mutually exclusive, mutations in $P B R M 1$ and SETD2 appear to synergize. ${ }^{45}$

The prognostic significance of many of these genes has been an area of active research. Kapur et $\mathrm{al}^{46}$ performed a retrospective analysis of 145 patients with ccRCC at The University of Texas Southwestern Medical Center, the majority of whom had localized or locoregional disease. Their results demonstrated that patients with tumors harboring BAPI mutations had significantly reduced median OS compared with those who had tumors containing PBRM1 mutations (4.6 vs. 10.6 years, respectively; HR $2.7 ; \mathrm{p}=.04$ ). ${ }^{46}$ They observed similar results in analysis of a second cohort (with 327 patients) from The Cancer Genome Atlas (TCGA). ${ }^{46}$ Hakimi et al ${ }^{47}$ performed a similar study looking at 188 patients who underwent resection of primary ccRCC at the Memorial Sloan Kettering Cancer Center, as well as an independent cohort of 421 patients from the TCGA. BAP1 mutations were associated with worse cancer-specific survival, with an HR of $7.71(\mathrm{p}=.002)$. PBRM1 mutations appeared to have no impact on cancer-specific survival, whereas SETD2 mutations were associated with worse cancer-specific survival only in the TCGA cohort (HR $1.68 ; \mathrm{p}=.036)$. 
Beyond single gene mutations, other studies have explored the use of gene expression profiling in the prognostication of RCC. Brannon et $\mathrm{al}^{48}$ obtained gene expression data from 48 ccRCC samples and identified two distinct molecular subtypes, which they defined as clear cell type A and clear cell type B. Using a validation data set of 177 samples, patients with clear cell type A tumors had significantly better cancer-specific survival than those with clear cell type B tumors (median survival, 8.6 vs. 2.0 years, respectively; $p=.002$ ). This signature was subsequently validated in a meta-analysis of six ccRCC gene expression data sets encompassing a total of 480 patients. ${ }^{49}$ Similarly, an analysis of the TCGA data set revealed four distinct molecular subgroups, designated $\mathrm{m} 1$ to $\mathrm{m} 4$. Similar to the study by Brannon et al, ${ }^{49}$ postnephrectomy survival was related to these ccRCC subtypes.

Interestingly, this study identified changes in key metabolic pathways within these subtypes. This included a more aggressive phenotype that was associated with increased expression of enzymes associated with the pentose phosphate shunt, glutamine transport, and fatty acid synthesis. ${ }^{43}$

\section{Predictive Biomarkers}

As alluded to previously, the use of molecular classifications to predict response to therapy is a crucial step toward a more personalized approach to the treatment of kidney cancer. With regard to VEGF-targeted therapy, a number of tumor-specific factors have been studied as potential biomarkers, including $V H L$ mutations and hypoxia-inducible factor levels. None of these are currently used in clinical practice and require further prospective validation. ${ }^{50}$ Interestingly, however, expression of the particular hypoxia-inducible factor $a$ isoform may predict responsiveness to a new class of agents targeting hypoxia-inducible factor $2 a{ }^{51}$

Beuselinck et $\mathrm{al}^{52}$ performed an integrated genomic analysis of primary ccRCCs to identify subgroups that may be more sensitive or resistant to anti-VEGF treatment. They collected primary tumor samples from 121 patients with metastatic ccRCC who were receiving firstline sunitinib. Using gene expression profiling, they identified four robust ccRCC subtypes (ccRCC1-ccRCC4). These groups showed a high correlation with the prognostic groups previously described by Brannon et al. ${ }^{48}$ These four molecular subtypes were associated with different responses to sunitinib treatment: $\mathrm{ccRCC} 1 / \mathrm{ccRCC} 4$ tumors had a lower response rate $(\mathrm{p}=.005)$ and a shorter PFS and OS compared with the ccRCC2/ccRCC3 subtypes ( $\mathrm{p}=.001$ and .0003 , respectively). The ccRCC4 subtype was associated with the poorest sunitinib response.

The poor-responder ccRCC1/ccRCC4 subtypes appeared to share a number of common molecular characteristics such as upregulation of MYC targets or a hypermethylated status strongly correlated with a polycomb stem-cell phenotype. The ccRCC 3 tumors revealed a gene expression profile similar to that of the normal kidney and appeared to have an indolent disease course. The ccRCC4 tumors showed specific pathologic features such as a more inflammatory and sarcomatoid phenotype as well as an upregulation of cellular immune pathways. These findings suggest that the ccRCC4 subtype may be more susceptible to immune-based therapies. The authors have subsequently shown that these four molecular subtypes are also associated with outcome among patients receiving pazopanib as first-line 
therapy. ${ }^{53}$ Although intriguing, the results must be further validated in a larger, independent patient cohort before they are used in routine clinical practice for treatment selection.

There have also been a number of studies examining potential predictive biomarkers to mTOR-directed therapy. Kucejova et $\mathrm{al}^{54}$ identified mutations in the mTOR-negative regulator TSC1 in ccRCC and proposed that such mutations may identify tumors most likely to respond to mTOR inhibitors. Kwiatkowski et al ${ }^{55}$ retrospectively analyzed a cohort of 79 patients with mRCC treated with mTOR inhibitors. They performed molecular genetic analysis on both responders and nonresponders to identify mutations associated with response. Mutations in MTOR, TSC1, or TSC2 were more common for patients who experienced clinical benefit than for those who progressed. However, a substantial fraction of responders ( 31 of $43 ; 72 \%$ ) had no mTOR pathway mutation identified. Thus, more research is needed before promising biomarkers such as these are used for treatment selection in clinical practice.

With regard to immune-based therapies, the use of high-dose IL-2 remains a first-line option for a select group of patients. Early evidence suggested that certain tumor characteristics such as carbonic anhydrase IX expression may predict for response. Unfortunately, the phase II SELECT trial (with 120 patients) failed to demonstrate the predictive value of carbonic anhydrase IX expression on overall response rate. ${ }^{56}$ The identification of reliable predictive biomarkers for immune checkpoint inhibitors is another area of active research in many tumor types, including RCC. The immune checkpoints most commonly targeted in cancer are the PD-1/PD-L1 pathway and the CTLA-4 pathway. In mRCC, the PD-1 inhibitor nivolumab has shown activity in the second-line setting, as well as in the first-line setting in combination with the CTLA-4 inhibitor, ipilimumab. ${ }^{28,39}$

One of the most studied potential biomarkers for immunotherapy is PD-L1 expression. Unlike in other tumors, PD-L1 has not been shown to be a reliable predictor of response to anti-PD-1 therapy in RCC. In the CheckMate 025 trial exploring nivolumab in the secondline setting, a benefit was observed with nivolumab irrespective of PD-L1 expression. ${ }^{39}$ In a subgroup analysis of the previously discussed Check-Mate 214 trial, patients with tumor PD-L1 of $1 \%$ or greater demonstrated a higher response rate and improved PFS with nivolumab and ipilimumab compared with sunitinib, but those with less than $1 \%$ expression still had a response rate of $37 \% .{ }^{28}$ IMmotion 151 is a recently reported randomized trial that compared bevacizumab and atezolizumab therapy to sunitinib, specifically in PD-L1 positive (> $1 \%$ expression on tumor infiltrating cells; SP142 assay) patients with metastatic renal cancer. ${ }^{57}$ The primary endpoint of the study was investigator assessed PFS in PD-L1 positive patients with untreated metastatic RCC and OS in all patients by intent to treat. The results revealed that PFS was significantly improved with the combination in PD-L1 positive patients (HR 0.74; 95\% CI, 0.57-0.96; $\mathrm{p}=.0217$ ). Median PFS was 7.7 months with sunitinib and 11.2 months with atezolizumab and bevacizumab combination. The OS results are not mature at the time of present report. In general, there are several key limitations with the use of PD-L1 expression as a potential biomarker. These include heterogeneity between the primary and metastatic sites as well as intratumor heterogeneity. PD-L1 is also a dynamic biomarker, which may change based on prior VEGF-targeted therapy. Other 
limitations involve the technical methods used, including the choice of antibody, the selected cutoffs to define positivity, and the types of cells analyzed. ${ }^{57}$

To explore genomic alterations in ccRCC that may correlate with response to anti-PD-1 therapy, Miao et al ${ }^{58}$ performed whole exome sequencing of metastatic ccRCC from 35 patients treated with nivolumab. In this analysis, they found that loss-of-function mutations in the PBRM1 gene appeared to be associated with increased clinical benefit to immune checkpoint therapy. Those with PBRM1 loss had significantly prolonged OS and PFS compared with patients without PBRM1 loss (log-rank $\mathrm{p}=.0074$ and $\mathrm{p}=.029$, respectively). These findings were confirmed in an independent validation cohort of 63 patients with ccRCC treated with checkpoint inhibitors. It is speculated that $P B R M 1$ loss may alter global expression profiles to influence responsiveness to immune-based therapies. The use of genomic analysis to predict response to immunotherapy represents an exciting step forward in the personalized care of $\mathrm{mRCC}$. These findings will need further prospective validation before they are used in clinical practice.

\section{Non-Clear Cell Renal Cell Carcinoma}

nccRCC represents approximately $20 \%$ of diagnoses and comprises a number of distinct histologies, each of which appears to have unique biology. The most common of the nonclear cell variants is papillary RCC. Durinck et al ${ }^{59}$ performed integrated genomic analyses of 167 non-clear cell tumors, including 67 papillary RCCs. The authors identified 10 significantly mutated genes, including $M E T, N F 2, S L C 5 A 3, P N K D$, and $C P Q .{ }^{59}$ The TCGA Network recently performed a comprehensive molecular characterization of papillary RCC from 161 samples and confirmed two clinically and biologically distinct subtypes. ${ }^{60}$ Type I disease was noted to bear a higher frequency of alterations in the $M E T$ proto-oncogene, whereas type II tumors had $C D K N 2 A$ silencing and SETD2 mutations. Data from the French RCC Network further support $M E T$ as an oncogenic driver across papillary RCC subtypes. In their analysis of 220 samples, $81 \%$ and $46 \%$ of type I and type II cases, respectively, demonstrated alterations in the MET gene. ${ }^{61}$

These findings have led to interest in exploring the use of MET-directed therapies in papillary RCC. The predictive ability of MET mutational status was demonstrated in a phase II trial looking at the MET inhibitor foretinib in metastatic papillary RCC. In this trial, the presence of a germline $M E T$ mutation was highly predictive of a response to this novel targeted therapy. ${ }^{62}$ Another phase II study evaluated the safety and efficacy of the MET inhibitor savolitinib for patients with papillary RCC according to MET status. ${ }^{63}$ In this trial, ORR was significantly higher for patients with MET-driven disease (18\% vs. none; $p=$. 002). Median PFS for patients with MET-driven and MET-independent disease was 6.2 and 1.4 months, respectively (HR $0.33 ; \mathrm{p}<.001$ ). Given these findings, an international, randomized, phase II study led by the Southwest Oncology Group (SWOG 1500) is further exploring this approach. This trial is randomly assigning patients with metastatic papillary RCC to receive either sunitinib or one of three MET-directed therapies: savolitinib, cabozantinib, and crizotinib. There will be an additional exploratory evaluation of MET mutational status and expression. The results of this trial could help change the treatment paradigm of nccRCC and further promote a more personalized approach to therapy. 


\section{Future Directions: ctDNA}

RCC is characterized by a high degree of intratumor heterogeneity. Analyses of RCC samples have shown that a single tumor biopsy may reveal only a minority of the genetic alterations within the entire tumor and that differences in genetic alterations are seen between the primary and metastatic sites. ${ }^{64,65}$ This degree of heterogeneity and clonal evolution complicates biomarker development and the delivery of precision medicine. Given these limitations, there has been increasing interest in the use ctDNA in kidney cancer.

Pal et $\mathrm{al}^{66}$ conducted the largest patient series of ctDNA evaluation in mRCC to date. In this study, they obtained ctDNA profiles from a cohort of 220 consecutive patients with mRCC. ${ }^{66}$ Genomic alterations were detected for $79 \%$ of patients. The most frequent alterations included TP53 (35\%), VHL (23\%), EGFR (17\%), NF1 (16\%), and ARID1A (12\%). They also attempted to define differences in the ctDNA profile across lines of targeted therapy. When looking at post first-line VEGF-therapy versus first-line VEGF-therapy profiles, they identified differences in genomic alterations: TP53 (64\% vs. 31\%; $\mathrm{p}=.04), N F 1$ (29\% vs. $4 \% ; \mathrm{p}=.02)$, and PIK3CA (29\% vs. $8 \% ; \mathrm{p}=.07)$. These changes may suggest a selective pressure from therapy and could imply a role in therapeutic resistance. Although it is not without a number of important limitations, this study illustrates the potential of ctDNA in further evaluating the genomic diversity of RCC. In the future, the use of ctDNA may expedite biomarker discovery and facilitate the selection of novel targeted therapies.

\section{CONCLUSION}

In summary, the treatment of advanced RCC has undergone an impressive transformation over the last decade. With a number of highly effective therapies available across multiple lines, it will become increasingly important to develop a more tailored approach to treatment selection. Clinical prognostic models like the IMDC have shown a predictive ability in the context of immune checkpoint inhibition in the first-line setting and will likely be used in clinical practice for patient selection. In Figure 1, we present a proposed plan based on current available information to help determine frontline therapy of ccRCC. As our understanding of the genomic landscape of RCC improves, a number of molecular markers are being explored as biomarkers. These include robust gene expression profiles that will hopefully further improve our ability to predict who will and will not respond to targeted therapy. Novel platforms such as ctDNA analysis may also provide a less-invasive avenue toward personalized medicine. In the end, given the complexity of cancer treatment, it will likely require a combination of clinical and biologic approaches to fully realize the potential of precision oncology.

\section{References}

1. Choueiri TK, Motzer RJ. Systemic therapy for metastatic renal-cell carcinoma. N Engl J Med 2017;376:354-366. [PubMed: 28121507]

2. Haddad AQ, Margulis V Tumour and patient factors in renal cell carcinoma-towards personalized therapy. Nat Rev Urol 2015;12:253-262. [PubMed: 25868564]

3. Malouf GG, Flippot R, Khayat D. Therapeutic strategies for patients with metastatic renal cell carcinoma in whom first-line vascular endothelial growth factor receptor-directed therapies fail. J Oncol Pract 2016;12:412-420. [PubMed: 27170687] 
4. Motzer RJ, Bacik J, Murphy BA, et al. Interferon-alfa as a comparative treatment for clinical trials of new therapies against advanced renal cell carcinoma. J Clin Oncol 2002;20:289-296. [PubMed: 11773181]

5. Heng DYC, Xie W, Regan MM, et al. Prognostic factors for overall survival in patients with metastatic renal cell carcinoma treated with vascular endothelial growth factor-targeted agents: results from a large, multicenter study. J Clin Oncol 2009;27:5794-5799. [PubMed: 19826129]

6. Heng DYC, Xie W, Regan MM, et al. External validation and comparison with other models of the International Metastatic Renal-Cell Carcinoma Database Consortium prognostic model: a population-based study. Lancet Oncol 2013;14:141-148. [PubMed: 23312463]

7. Ko JJ, Xie W, Kroeger N, et al. The International Metastatic Renal Cell Carcinoma Database Consortium model as a prognostic tool in patients with metastatic renal cell carcinoma previously treated with first-line targeted therapy: a population-based study. Lancet Oncol 2015;16:293-300. [PubMed: 25681967]

8. Wells JC, Stukalin I, Norton C, et al. Third-line targeted therapy in metastatic renal cell carcinoma: results from the International Metastatic Renal Cell Carcinoma Database Consortium. Eur Urol 2017;71:204-209. [PubMed: 27318422]

9. Kroeger N, Xie W, Lee JL, et al. Metastatic non-clear cell renal cell carcinoma treated with targeted therapy agents: characterization of survival outcome and application of the International mRCC Database Consortium criteria. Cancer. 2013;119:2999-3006. [PubMed: 23696129]

10. Yip S, Wells C, Moreira RB, et al. Real world experience of immuno-oncology agents in metastatic renal cell carcinoma: results from the IMDC. J Clin Oncol 2017;35 (suppl; abstr 492).

11. Pérez-Valderrama B, Arranz Arija JA, Rodríguez Sánchez A, et al. Validation of the International Metastatic Renal-Cell Carcinoma Database Consortium (IMDC) prognostic model for first-line pazopanib in metastatic renal carcinoma: the Spanish Oncologic Genitourinary Group (SOGUG) SPAZO study. Ann Oncol 2016;27:706-711. [PubMed: 26658889]

12. McKay RR, Kroeger N, Xie W, et al. Impact of bone and liver metastases on patients with renal cell carcinoma treated with targeted therapy. Eur Urol 2014;65:577-584. [PubMed: 23962746]

13. Templeton AJ, Knox JJ, Lin X, et al. Change in neutrophil-to-lymphocyte ratio in response to targeted therapy for metastatic renal cell carcinoma as a prognosticator and biomarker of efficacy. Eur Urol 2016;70:358-364. [PubMed: 26924770]

14. Khambati HK, Choueiri TK, Kollmannsberger CK, et al.; International mRCC Database Consortium. Efficacy of targeted therapy for metastatic renal cell carcinoma in the elderly patient population. Clin Genitourin Cancer. 2014;12:354-358. [PubMed: 24819320]

15. Mitchell AP, Hirsch BR, Harrison MR, et al. Deferred systemic therapy in patients with metastatic renal cell carcinoma. Clin Genitourin Cancer. 2015;13:e159-e166. [PubMed: 25770767]

16. Harrison MR, Bhavsar NA, Wolf SP, et al. Deferred systemic therapy (DST) in the prospective metastatic renal cell cancer (MaRCC) registry. J Clin Oncol 2016;34 (suppl; abstr e16084).

17. Park I, Lee JL, Ahn JH, et al. Active surveillance for metastatic or recurrent renal cell carcinoma. J Cancer Res Clin Oncol 2014;140:1421-1428. [PubMed: 24752340]

18. Rini BI, Dorff TB, Elson P, et al. Active surveillance in metastatic renal-cell carcinoma: a prospective, phase 2 trial. Lancet Oncol 2016;17:1317-1324. [PubMed: 27498080]

19. Bianchi M, Sun M, Jeldres C, et al. Distribution of metastatic sites in renal cell carcinoma: a population-based analysis. Ann Oncol 2012;23:973-980. [PubMed: 21890909]

20. Kavolius JP, Mastorakos DP, Pavlovich C, et al. Resection of metastatic renal cell carcinoma. J Clin Oncol 1998;16:2261-2266. [PubMed: 9626229]

21. Pfannschmidt J, Hoffmann H, Muley T, et al. Prognostic factors for survival after pulmonary resection of metastatic renal cell carcinoma. Ann Thorac Surg 2002;74:1653-1657. [PubMed: 12440625]

22. Tanis PJ, van der Gaag NA, Busch ORC, et al. Systematic review of pancreatic surgery for metastatic renal cell carcinoma. Br J Surg 2009;96:579-592. [PubMed: 19434703]

23. Wang CJ, Christie A, Lin MH, et al. Safety and efficacy of stereotactic ablative radiation therapy for renal cell carcinoma extracranial metastases. Int J Radiat Oncol Biol Phys 2017;98:91-100. [PubMed: 28587057] 
24. Aoun HD, Littrup PJ, Jaber M, et al. Percutaneous cryoablation of renal tumors: is it time for a new paradigm shift? J Vasc Interv Radiol 2017;28:1363-1370. [PubMed: 28844831]

25. Mehta S, Illidge T, Choudhury A. Immunotherapy with radiotherapy in urological malignancies. Curr Opin Urol 2016;26:514-522. [PubMed: 27552139]

26. Hudes G, Carducci M, Tomczak P, et al.; Global ARCC Trial. Temsirolimus, interferon alfa, or both for advanced renal-cell carcinoma. N Engl J Med 2007;356:2271-2281. [PubMed: 17538086]

27. Choueiri TK, Halabi S, Sanford BL, et al. Cabozantinib versus sunitinib as initial targeted therapy for patients with metastatic renal cell carcinoma of poor or intermediate risk: the Alliance A031203 CABOSUN trial. J Clin Oncol 2017;35:591-597. [PubMed: 28199818]

28. Escudier B, Tannir NM, McDermott DF, et al. CheckMate 214: efficacy and safety of nivolumab + ipilimumab (N+I) v sunitinib (S) for treatment-naïve advanced or metastatic renal cell carcinoma (mRCC), including IMDC risk and PD-L1 expression subgroups. Ann Oncol 2017;28 (suppl; abstr LBA5).

29. Flanigan RC, Mickisch G, Sylvester R, et al. Cytoreductive nephrectomy in patients with metastatic renal cancer: a combined analysis. J Urol 2004;171:1071-1076. [PubMed: 14767273]

30. Heng DYC, Wells JC, Rini BI, et al. Cytoreductive nephrectomy in patients with synchronous metastases from renal cell carcinoma: results from the International Metastatic Renal Cell Carcinoma Database Consortium. Eur Urol 2014;66:704-710. [PubMed: 24931622]

31. Bex A, Mulders P, Jewett MAS, et al. Immediate versus deferred cytoreductive nephrectomy (CN) in patients with synchronous metastatic renal cell carcinoma (mRCC) receiving sunitinib (EORTC 30073 SURTIME). Ann Oncol 2017;28 (suppl; abstr LBA35).

32. Kollmannsberger C. Sunitinib side effects as surrogate biomarkers of efficacy. Can Urol Assoc J. 2016;10(suppl 7):S245-S247. [PubMed: 28096937]

33. Donskov F, Michaelson MD, Puzanov I, et al. Sunitinib-associated hypertension and neutropenia as efficacy biomarkers in metastatic renal cell carcinoma patients. Br J Cancer. 2015;113:1571-1580. [PubMed: 26492223]

34. Motzer RJ, Hutson TE, Tomczak P, et al. Sunitinib versus interferon alfa in metastatic renal-cell carcinoma. N Engl J Med 2007;356:115-124. [PubMed: 17215529]

35. Rini BI, Cohen DP, Lu DR, et al. Hypertension as a biomarker of efficacy in patients with metastatic renal cell carcinoma treated with sunitinib. J Natl Cancer Inst 2011;103:763-773. [PubMed: 21527770]

36. Al-Marrawi MY, Rini BI, Harshman LC, et al.; International mRCC Database Consortium. The association of clinical outcome to first-line VEGF-targeted therapy with clinical outcome to second-line VEGF-targeted therapy in metastatic renal cell carcinoma patients. Target Oncol 2013;8:203-209. [PubMed: 23300029]

37. Choueiri TK, Escudier B, Powles T, et al.; METEOR Investigators. Cabozantinib versus everolimus in advanced renal-cell carcinoma. N Engl J Med 2015;373:1814-1823. [PubMed: 26406150]

38. Escudier B, Powles T, Motzer RJ, et al. Cabozantinib, a new standard of care for patients with advanced renal cell carcinoma and bone metastases? Subgroup analysis of the METEOR trial. J Clin Oncol Epub 201818.

39. Motzer RJ, Escudier B, McDermott DF, et al.; CheckMate 025 Investigators. Nivolumab versus everolimus in advanced renal-cell carcinoma. N Engl J Med 2015;373:1803-1813. [PubMed: 26406148]

40. Escudier B, Sharma P, McDermott DF, et al.; CheckMate 025 Investigators. CheckMate 025 randomized phase 3 study: outcomes by key baseline factors and prior therapy for nivolumab versus everolimus in advanced renal cell carcinoma. Eur Urol 2017;72:962-971. [PubMed: 28262413]

41. Jeyakumar G, Kim S, Bumma N, et al. Neutrophil lymphocyte ratio and duration of prior antiangiogenic therapy as biomarkers in metastatic RCC receiving immune checkpoint inhibitor therapy. J Immunother Cancer. 2017;5:82. [PubMed: 29041991]

42. Srigley JR, Delahunt B, Eble JN, et al.; ISUP Renal Tumor Panel. The International Society of Urological Pathology (ISUP) Vancouver Classification of Renal Neoplasia. Am J Surg Pathol 2013;37:1469-1489. [PubMed: 24025519] 
43. Cancer Genome Atlas Research Network. Comprehensive molecular characterization of clear cell renal cell carcinoma. Nature. 2013; 499:43-49. [PubMed: 23792563]

44. Sato Y, Yoshizato T, Shiraishi Y, et al. Integrated molecular analysis of clear-cell renal cell carcinoma. Nat Genet 2013;45:860-867. [PubMed: 23797736]

45. Pena-Llopis S, Christie A, Xie X-J, et al. Cooperation and antagonism among cancer genes: the renal cancer paradigm. Cancer Res 2013;73:4173-4179. [PubMed: 23832661]

46. Kapur P, Pena-Llopis S, Christie A, et al. Effects on survival of BAP1 and PBRM1 mutations in sporadic clear-cell renal-cell carcinoma: a retrospective analysis with independent validation. Lancet Oncol 2013;14:159-167. [PubMed: 23333114]

47. Hakimi AA, Ostrovnaya I, Reva B, et al. Adverse outcomes in clear cell renal cell carcinoma with mutations of 3p21 epigenetic regulators BAP1 and SETD2: a report by MSKCC and the KIRC TCGA Research Network. Clin Cancer Res 2013;19:3259-3267. [PubMed: 23620406]

48. Brannon AR, Reddy A, Seiler M, et al. Molecular stratification of clear cell renal cell carcinoma by consensus clustering reveals distinct subtypes and survival patterns. Genes Cancer. 2010;1:152163. [PubMed: 20871783]

49. Brannon AR, Haake SM, Hacker KE, et al. Meta-analysis of clear cell renal cell carcinoma gene expression defines a variant subgroup and identifies gender influences on tumor biology. Eur Urol 2012;61:258-268. [PubMed: 22030119]

50. Sonpavde G, Choueiri TK. Biomarkers: the next therapeutic hurdle in metastatic renal cell carcinoma. Br J Cancer. 2012;107:1009-1016. [PubMed: 22948724]

51. Chen W, Hill H, Christie A, et al. Targeting renal cell carcinoma with a HIF-2 antagonist. Nature. 2016;539:112-117. [PubMed: 27595394]

52. Beuselinck B, Job S, Becht E, et al. Molecular subtypes of clear cell renal cell carcinoma are associated with sunitinib response in the metastatic seffing. Clin Cancer Res 2015;21:1329-1339. [PubMed: 25583177]

53. Verbiest A, Couchy G, Job S, et al. Molecular subtypes of clear cell renal cell carcinoma are associated with outcome during pazopanib therapy in the metastatic seffing. Clin Genitourin Cancer. Epub 2017117.

54. Kucejova B, Pena-Llopis S, Yamasaki T, et al. Interplay between pVHL and mTORC1 pathways in clear-cell renal cell carcinoma. Mol Cancer Res 2011;9:1255-1265. [PubMed: 21798997]

55. Kwiatkowski DJ, Choueiri TK, Fay AP, et al. Mutations in TSC1, TSC2, and MTOR are associated with response to rapalogs in patients with metastatic renal cell carcinoma. Clin Cancer Res 2016;22:2445-2452. [PubMed: 26831717]

56. McDermott DF, Cheng SC, Signoreffi S, et al. The high-dose aldesleukin "select" trial: a trial to prospectively validate predictive models of response to treatment in patients with metastatic renal cell carcinoma. Clin Cancer Res 2015;21:561-568. [PubMed: 25424850]

57. Mazza C, Escudier B, Albiges L. Nivolumab in renal cell carcinoma: latest evidence and clinical potential. Ther Adv Med Oncol 2017;9:171-181. [PubMed: 28344662]

58. Miao D, Margolis CA, Gao W, et al. Genomic correlates of response to immune checkpoint therapies in clear cell renal cell carcinoma. Science. 2018;59:801-806.

59. Durinck S, Stawiski EW, Pavia-Jimenez A, et al. Spectrum of diverse genomic alterations define non-clear cell renal carcinoma subtypes. Nat Genet 2015;47:13-21. [PubMed: 25401301]

60. Linehan WM, Spellman PT, Ricketts CJ, et al.; Cancer Genome Atlas Research Network. Comprehensive molecular characterization of papillary renal-cell carcinoma. N Engl J Med 2016;374:135-145. [PubMed: 26536169]

61. Albiges L, Guegan J, Le Formal A, et al. MET is a potential target across all papillary renal cell carcinomas: result from a large molecular study of pRCC with CGH array and matching gene expression array. Clin Cancer Res 2014;20:3411-3421. [PubMed: 24658158]

62. Choueiri TK, Vaishampayan U, Rosenberg JE, et al. Phase II and biomarker study of the dual MET/VEGFR2 inhibitor foretinib in patients with papillary renal cell carcinoma. J Clin Oncol 2013;31:181-186. [PubMed: 23213094]

63. Choueiri TK, Plimack E, Arkenau HT, et al. Biomarker-based phase II trial of savolitinib in patients with advanced papillary renal cell cancer J Clin Oncol 2017;35:2993-3001. [PubMed: 28644771] 
64. Gerlinger M, Horswell S, Larkin J, et al. Genomic architecture and evolution of clear cell renal cell carcinomas defined by multiregion sequencing. Nat Genet 2014;46:225-233. [PubMed: 24487277]

65. Gerlinger M, Rowan AJ, Horswell S, et al. Intratumor heterogeneity and branched evolution revealed by multiregion sequencing. N Engl J Med 2012;366:883-892. [PubMed: 22397650]

66. Pal SK, Sonpavde G, Agarwal N, et al. Evolution of circulating tumor DNA profile from first-line to subsequent therapy in metastatic renal cell carcinoma. Eur Urol 2017;72:557-564. [PubMed: 28413127] 


\section{PRACTICAL APPLICATIONS}

- As the number of therapeutic options in metastatic renal cell carcinoma increases, it will be critical to develop a more personalized strategy to treatment, taking into consideration both tumor and patient characteristics to develop a tailored treatment plan.

- $\quad$ Clinical prognostic models like the IMDC in advanced renal cell carcinoma are important tools for both clinical decision making and risk stratification in clinical trials.

- More robust predictors of response to novel immuno-oncology agents, including immune checkpoint inhibitors, are an unmet need in metastatic renal cell carcinoma.

- Modern genomic profiling in renal cell carcinoma is uncovering a number of promising predictive and prognostic molecular biomarkers.

- $\quad$ Enrollment of patients with advanced kidney cancer in biomarker-directed clinical trials is encouraged. 


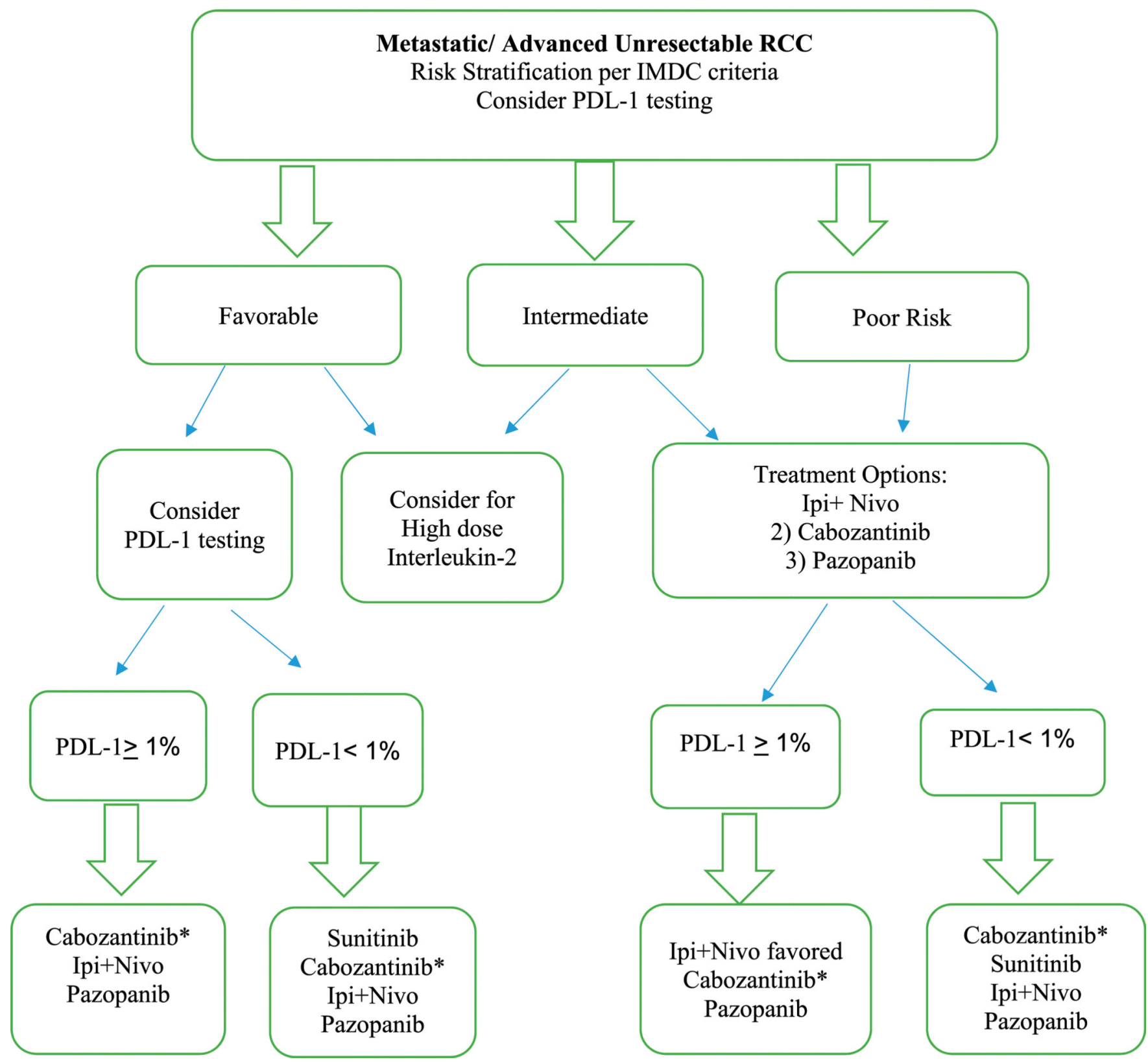

FIGURE 1. Personalized Frontline Therapy of Renal Cell Carcinoma

Asterisks indicate that cabozantinib should be considered for patients with bone metastases. The recommendation for PD-L1 testing for treatment decisions is based on subset analysis of CheckMate 214.

Abbreviations: RCC, renal cell carcinoma; IMDC, International Metastatic Renal Cell Carcinoma Database Consortium; Ipi+Nivo, ipilimumab/nivolumab. 
TABLE 1.

IMDC and MSKCC Prognostic Models

\begin{tabular}{|c|c|c|}
\hline Prognostic Factor & Risk Groups & $\begin{array}{l}\text { Median OS } \\
\text { (Months) }\end{array}$ \\
\hline \multicolumn{3}{|l|}{$\mathrm{IMDC}^{5}$} \\
\hline Low Karnofsky performance $(<80 \%)$ & Favorable risk (0 factors) & 43 \\
\hline \multicolumn{3}{|l|}{ Time from diagnosis to treatment $<1$ year } \\
\hline Low hemoglobin $(<\mathrm{LLN})$ & Intermediate risk ( $1-2$ factors) & 23 \\
\hline \multicolumn{3}{|l|}{ High corrected calcium (> ULN) } \\
\hline High neutrophils (> ULN) & Poor risk ( $\geq 3$ factors) & 8 \\
\hline \multicolumn{3}{|l|}{ High platelets (> ULN) } \\
\hline \multicolumn{3}{|l|}{$\mathrm{MSKCC}^{4}$} \\
\hline Low Karnofsky performance $(<80 \%)$ & Favorable risk (0 factors) & 30 \\
\hline \multicolumn{3}{|l|}{ Time from diagnosis to treatment $<1$ year } \\
\hline Low hemoglobin $(<\mathrm{LLN})$ & Intermediate risk ( $1-2$ factors) & 14 \\
\hline \multicolumn{3}{|l|}{ High corrected calcium (> 10 mg/dL) } \\
\hline High LDH (> 1.5 times ULN) & Poor risk ( 23 factors) & 5 \\
\hline
\end{tabular}

Abbreviations: IMDC, International Metastatic Renal Cell Carcinoma Database Consortium; MSKCC, Memorial Sloan Kettering Cancer Center; OS, overall survival; LLN, lower limit of normal; ULN, upper limit of normal; LDH, lactate dehydrogenase. 


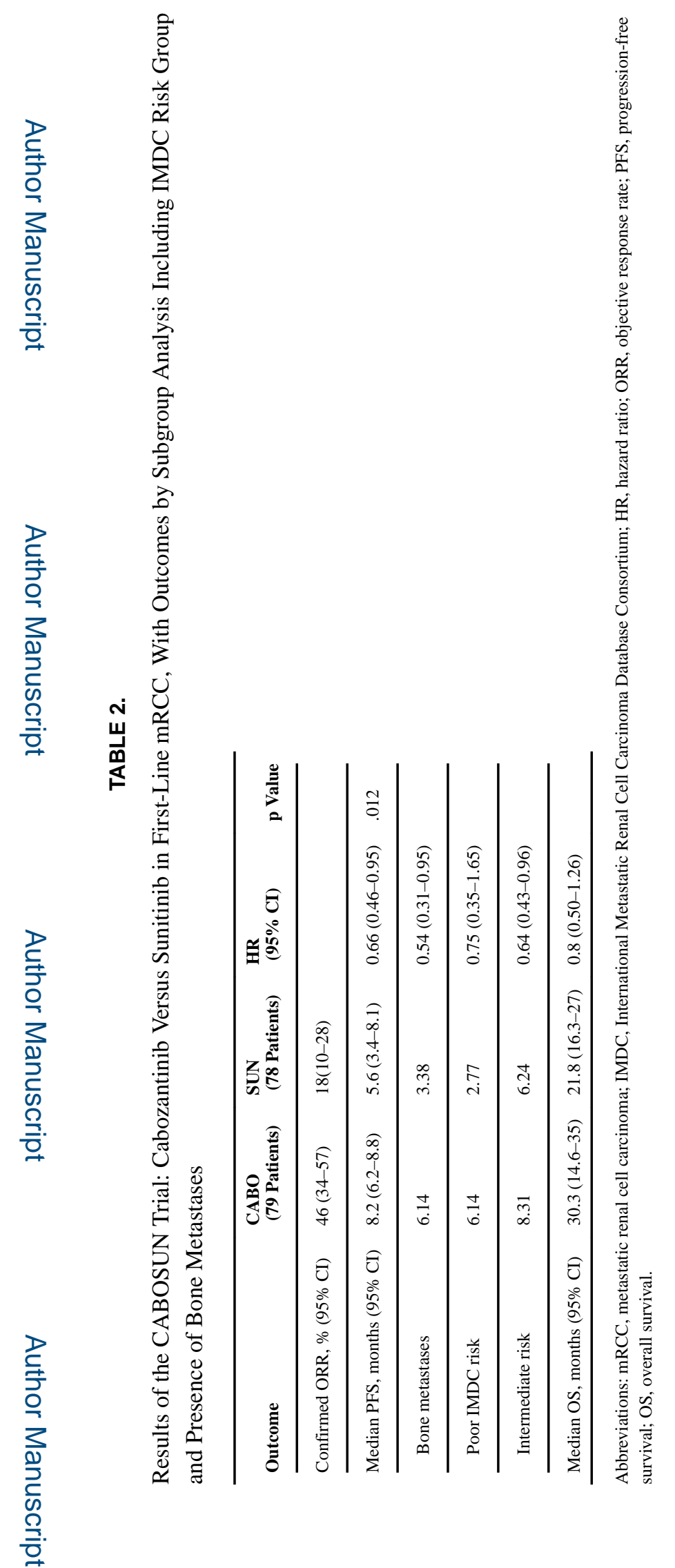

Am Soc Clin Oncol Educ Book. Author manuscript; available in PMC 2019 November 20. 

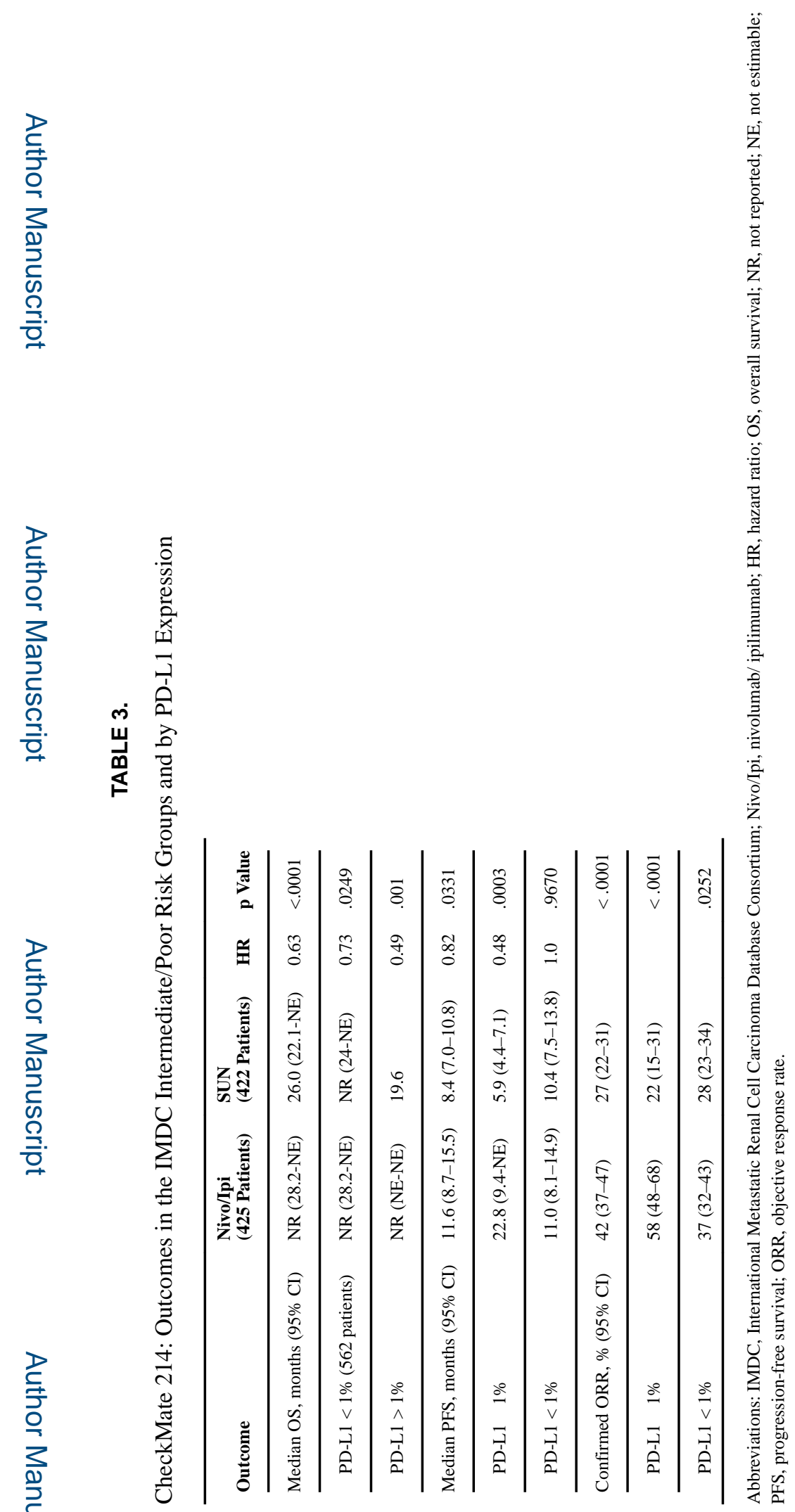